\title{
Probing the Epoch of Reionization with the Lya Forest at Z \# 4-5
}

\section{Citation}

Cen, Renyue, Patrick McDonald, Hy Trac, and Abraham Loeb. 2009. "PROBING THE EPOCH OF REIONIZATION WITH THE Lya FOREST ATz\# 4-5.” The Astrophysical Journal 706 (1): L164-67. https://doi.org/10.1088/0004-637x/706/1//164.

\section{Permanent link}

http://nrs.harvard.edu/urn-3:HUL.InstRepos:41412176

\section{Terms of Use}

This article was downloaded from Harvard University's DASH repository, and is made available under the terms and conditions applicable to Other Posted Material, as set forth at http:// nrs.harvard.edu/urn-3:HUL.InstRepos:dash.current.terms-of-use\#LAA

\section{Share Your Story}

The Harvard community has made this article openly available.

Please share how this access benefits you. Submit a story.

Accessibility 


\title{
PROBING THE EPOCH OF REIONIZATION WITH THE Ly $\alpha$ FOREST AT $z \sim 4-5$
}

\author{
Renyue Cen ${ }^{1}$, Patrick McDonald $^{2}$, Hy Trac $^{3}$, And Abraham Loeb ${ }^{3}$ \\ ${ }^{1}$ Department of Astrophysical Sciences, Princeton University, Princeton, NJ 08544, USA \\ ${ }^{2}$ Canadian Institute for Theoretical Astrophysics, University of Toronto, Toronto, ON M5S 3H8, Canada \\ ${ }^{3}$ Harvard-Smithsonian Center for Astrophysics, Cambridge, MA 02138, USA \\ Received 2009 July 16; accepted 2009 October 21; published 2009 November 4
}

\begin{abstract}
The inhomogeneous cosmological reionization process leaves tangible imprints in the intergalactic medium (IGM) down to $z \sim 4-5$. The Ly $\alpha$ forest flux power spectrum provides a potentially powerful probe of the epoch of reionization. With the existing Sloan Digital Sky Survey I/II quasar sample, we show that two cosmological reionization scenarios, one completing reionization at $z=6$ and the other at $z=9$, can be distinguished at $\sim 7 \sigma$ level by utilizing $\operatorname{Ly} \alpha$ forest absorption spectra at $z=3.9-4.1$ in the absence of other physical processes that may also affect the Ly $\alpha$ flux power spectrum. The difference may not be distinguishable at such high significance after marginalization over other effects, but, in any case, one will need to consider this effect in order to correctly interpret the power spectrum in this redshift range. The redshift range $z=4-5$ may provide the best window because there are still enough transmitted flux and quasars to measure precise statistics of the flux fluctuations, and the IGM still retains a significant amount of memory of reionization.
\end{abstract}

Key words: galaxies: high-redshift - hydrodynamics - intergalactic medium - large-scale structure of universe quasars: absorption lines - radiative transfer

\section{INTRODUCTION}

The history of cosmological reionization is presently primarily constrained by the cosmic microwave background observations of Wilkinson Microwave Anisotropy Probe (WMAP; Dunkley et al. 2009) and Sloan Digital Sky Survey (SDSS) quasar absorption spectra. The former gives an integral constraint, strongly suggesting that cosmological reionization may well be underway at $z \sim 12$, while the latter provides a solid anchor point at $z \sim 6$ when the universe became largely transparent to Lyman limit photons (e.g., Fan et al. 2001, 2006b; Becker et al. 2001; Cen \& McDonald 2002). At $z \geqslant 6.3$, the lower bound on the neutral hydrogen fraction, $x$, of the intergalactic medium (IGM) provided by SDSS observations is, however, fairly loose at $x \geqslant 0.01$. Thus, exactly when most of the neutral hydrogen became reionized is yet unknown and there are many possible scenarios that could meet the current observational constraints (e.g., Barkana \& Loeb 2001; Cen 2003; Haiman \& Holder 2003; Fan et al. 2006a; Wyithe \& Cen 2007; Becker et al. 2007).

The process of inhomogeneous cosmological reionization leaves quantifiable and significant imprints on the thermal evolution of the IGM (Trac et al. 2008; Furlanetto \& Oh 2009). In this Letter, we show that the $\operatorname{Ly} \alpha$ forest flux spectrum at moderate redshift $z=4.5 \pm 0.5$ sensitively depends on the epoch of reionization.

\section{REIONIZATION MODELS}

We use a hybrid code to accurately compute the reionization process, which consists of a high-resolution $\mathrm{N}$-body code, a shock-capturing total variation diminishing hydro code and a ray-tracing radiative transfer code. The reader is referred to Trac et al. (2008) for more details. The best-fit WMAP five-year cosmological parameters are used: $\Omega_{m}=0.28, \Omega_{\Lambda}=0.72$, $\Omega_{b}=0.046, h=0.70, \sigma_{8}=0.82$, and $n_{s}=0.96$ (Komatsu et al. 2009). We use 24 billion dark matter particles on an effective mesh with $11,520^{3}$ cells in a comoving box of $100 \mathrm{~h}^{-1} \mathrm{Mpc}$, yielding a particle mass resolution of $2.68 \times 10^{6} h^{-1} M_{\odot}$ and allowing us to resolve all atomic cooling dark matter halos. A total of $N=1536^{3}$ gas cells of size $65 \mathrm{~h}^{-1} \mathrm{kpc}$ are used, and we trace five frequency bins at $>13.6 \mathrm{eV}$ with the ray-tracing code. The star formation rate is controlled by the halo formation history. We adjust the ionizing photon escape fraction to arrive at two models, where reionization is completed early $(z \sim 9)$ and late $(z \sim 6)$, respectively.

\section{RESULTS}

Previous studies (e.g., Furlanetto et al. 2004; Iliev et al. 2006; Lee et al. 2008) have shown that the reionization process proceeds in an inside-out fashion, where regions around high-density peaks get reionized first. H II regions initially surround isolated galaxies that formed in high-density peaks. With time these $\mathrm{H}$ II regions expand and lower density (void) regions are eventually engulfed by the expanding H II regions stemming from high-density peaks. Consequently, the redshift of reionization of each individual spatial point, $z_{\mathrm{ri}}$, is highly correlated with the underlying large-scale density field, as we have shown earlier (Trac et al. 2008). Once an expanding region is photoionized and photoheated, it would cool subsequently due to adiabatic expansion and other cooling processes (primarily Compton cooling at high redshift), countered by photoheating of residual recombining hydrogen atoms (on the timescale of recombination; e.g., Theuns et al. 2002; Hui \& Haiman 2003). As a result, the strong correlation between $z_{\text {ri }}$ and the underlying large-scale density is manifested in a strong anti-correlation between the temperature and the underlying large-scale density field. Specifically, different regions of the same low densities $\delta \leqslant$ a few (without large-scale smoothing in this case) would display a large, long-range-correlated, dispersion in temperature, following the completion of reionization (e.g., Trac et al. 2008).

Both the anti-correlation between temperature and the underlying large-scale density and the consequent temperature dispersion at a fixed density weaken as time progresses and 

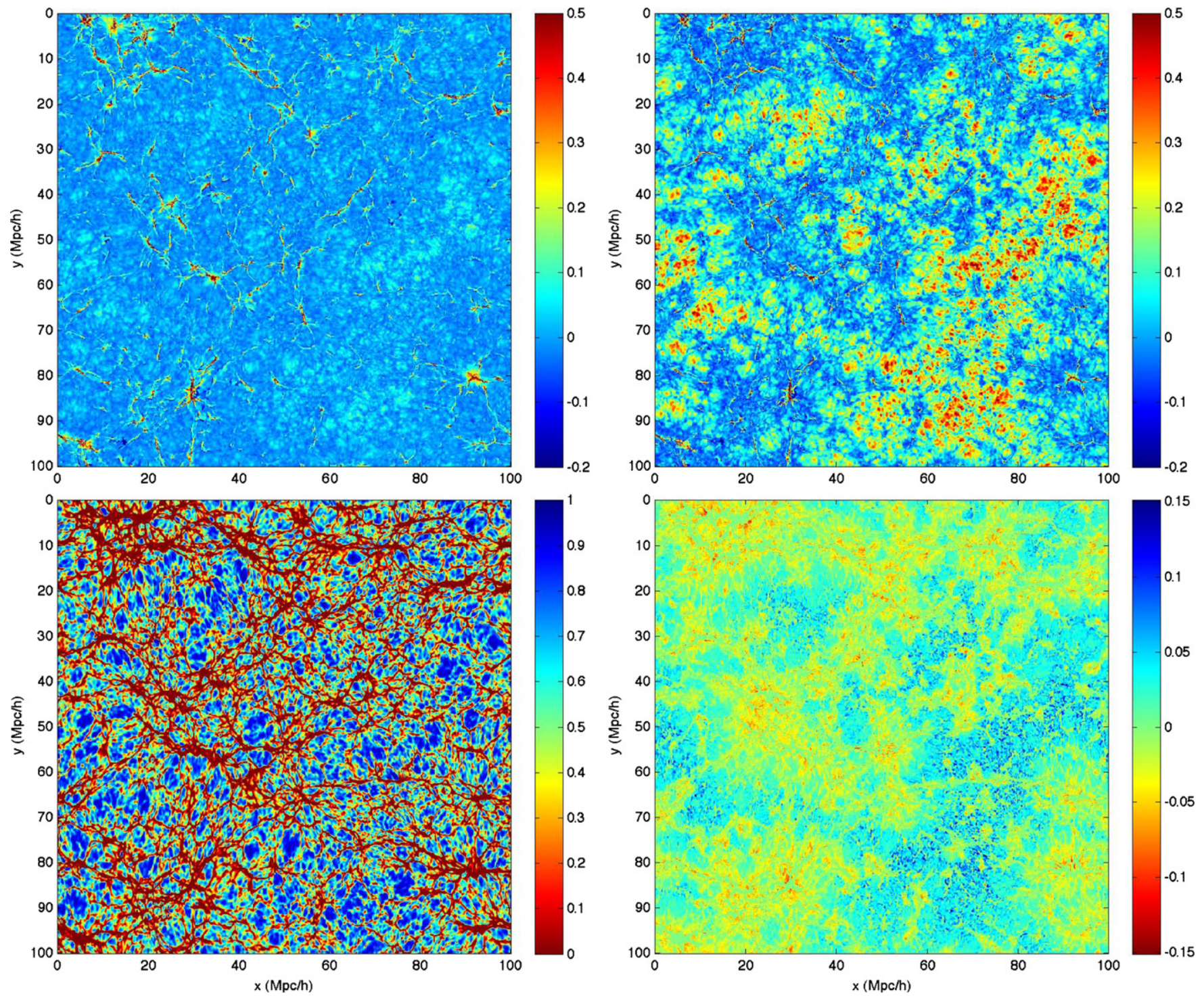

Figure 1. Top panels show the $\log$ of the ratio of gas temperature from the simulation to that prescribed by a fixed EoS at $z=4$, for the early (left) and late (right) reionization model, respectively. We use EoS formula $T=T_{0}\left(\rho / \rho_{0}\right)^{0.62}$, where $T_{0}$ is the temperature at mean density $\rho_{0}$ in each model. The slice shown has a size $\left(100 h^{-1} \mathrm{Mpc}\right)^{2}$ with a thickness equal to two hydro cells $\left(130 h^{-1} \mathrm{kpc}\right)$. The distribution of flux transmission, $F(\operatorname{early})=\exp (-\tau(\operatorname{early}))$, for the late-reionization model is shown in the bottom left, where $\tau$ (early) is the Ly $\alpha$ optical depth computed based on the distribution of neutral hydrogen density, gas peculiar velocity, and temperature at $z=4$. In computing the neutral hydrogen fraction, we have used a uniform background radiation field with its amplitude adjusted such that both models yield the same mean transmitted flux of $\langle F\rangle=0.43$ at $z=4$, as observed (Fan et al. 2006b). The flux difference between the two models: $F$ (late) $-F$ (early) $=$ $\exp (-\tau$ (late $))-\exp (-\tau$ (early $))$ is shown in the bottom right panel.

the temperature-density relation asymptotically approaches a so-called equation-of-state (EoS), a one-to-one mapping from IGM density to temperature (Hui \& Gnedin 1997), with $T=$ $T_{0}\left(\rho / \rho_{0}\right)^{0.62}$ in the late-time limit. However, at the redshift range $z=4.5 \pm 0.5$, the IGM has not had enough time to have completely relaxed to this asymptotic $\mathrm{EoS}(\mathrm{aEoS})$, or any one-to-one EoS, if the universe was reionized, say, at $z_{r i} \sim 6-8$. The deviations from a simple temperature-density relation are larger for smaller $z_{r i}$ at a given observed redshift.

In Figure 1, we show the log of the ratio of gas temperature from the simulation to that prescribed by the aEoS at $z=4$ in a slice of size $\left(100 h^{-1} \mathrm{Mpc}\right)^{2}$ with a thickness equal to two hydro cells $\left(130 h^{-1} \mathrm{kpc}\right.$ ), for the early (top left panel) and late (top right panel) reionization model, respectively. The small reddish/yellowish regions seen in the top left panel correspond to virialized regions, for which the plotted ratio does not contain useful information. But these regions show clearly the location of ionizing sources. We see striking differences in temperature distributions between the two reionization models with respect to their respective $\mathrm{aEoS}$ values. In the earlyreionization model (top left panel), most of the regions have blue color (i.e., the ratio equal to $\sim 1$ ) and appear to have mostly relaxed to the state predicted by the aEoS, while some low-density regions in the voids still display yellowish color with a temperature that is higher than that of the aEoS by $30 \%-50 \%$. On the other hand, in the late-reionization simulation (top right panel), while regions just outside the shock-heated filaments and halos (bluish color) have largely relaxed to the aEoS, regions of comparable local densities in the voids are much hotter than that of the aEoS, by a factor of 1.5-2.5.

Because the neutral hydrogen fraction in regions of moderate density is determined by the balance between photoionization rate and recombination rate, the latter of which is a function of 


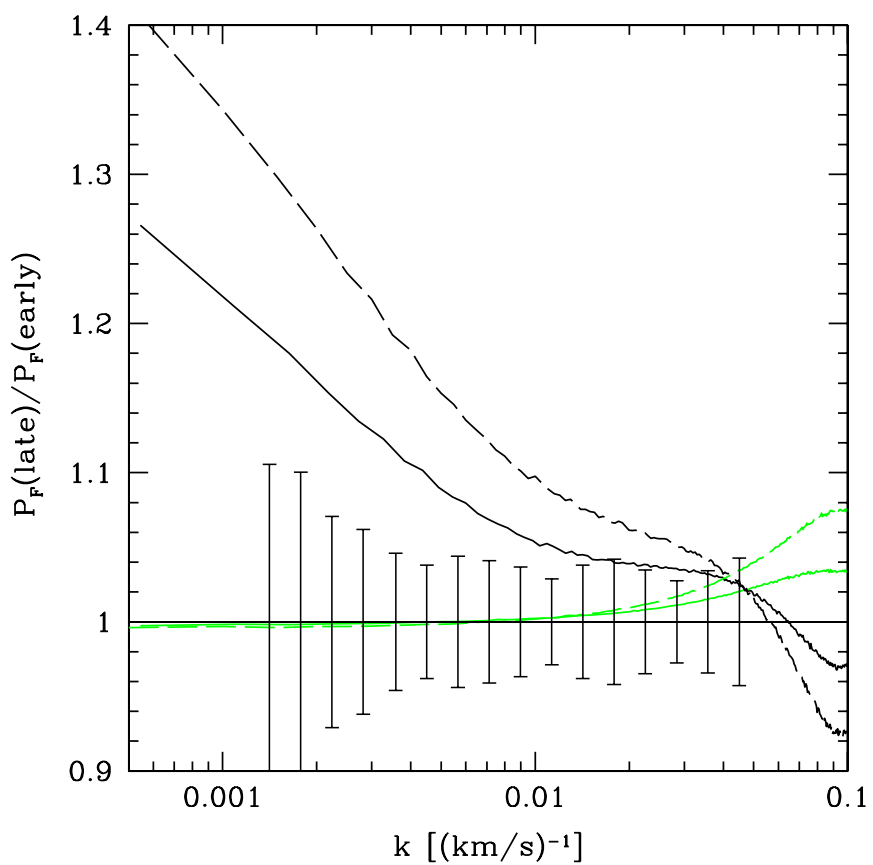

Figure 2. Black solid and dashed curves are the ratio of flux power spectrum in the late-reionization model to that in the early-reionization model at $z=4$ and $z=5$, respectively. Also shown as the two green curves are the corresponding ratios produced by replacing the real temperature in each simulation by that prescribed by the EoS given density (the same EoS in both simulations). The black error bars are the error one can expect from the full SDSS I/II sample plus existing high-resolution data. The error bars will be approximately uncorrelated. A formal analysis of the 16 data points indicates that the two reionization models can be differentiated at $7 \sigma$ level.

temperature, the two different temperature distributions in the two reionization models result in different large-scale neutral hydrogen distribution. In the bottom left panel of Figure 1, we show the expected flux transmission for the early-reionization model. In the bottom right panel, the flux difference between the two models is shown. It is seen that the transmitted Ly $\alpha$ flux is significantly affected by the temperature difference at $z=4$, resulting in fractional difference in the transmitted flux in the voids between the two models of $\sim 15 \%$ (blue regions). Specifically, there is more transmitted flux in the void regions in the late-reionization model, compensated by reduced transmission in high-density regions. It is noted that, at $z \geqslant 4$, the majority of transmitted $\operatorname{Ly} \alpha$ flux comes from the lowest density regions of $\delta \leqslant$ a few.

Figure 2 shows the ratio of flux power spectrum in the latereionization model to that in the early-reionization model at $z=4$ (black solid) and $z=5$ (black dashed). The large-scale anti-correlation between density and deviations from a single EoS in the late-reionization model leads to a significant amount of extra power in the flux spectrum (specifically, relatively high temperatures in late-reionizing underdense regions lead them to produce even less absorption than they otherwise would). The difference between the flux power spectra of the two reionization models increases with scale, reaching $20 \%$ at $k=$ $0.001\left(\mathrm{~km} \mathrm{~s}^{-1}\right)^{-1}$ at $z=4$; the difference is still larger at $z=5$ ( $\sim 30 \%)$, as expected.

The black error bars in Figure 2 indicate the statistical errors expected with the full SDSS I/II sample (completed, but not yet fully analyzed) in the redshift range $z=3.9-4.1$, augmented by (roughly) what one could obtain in a joint analysis of existing higher resolution data in the redshift range $z=3.9-4.1$. Specifically, the SDSS errors are a factor of $\sqrt{3}$ smaller than those for the redshift range $3.9<z<4.1$ in McDonald et al. (2006), representing a straightforward $\sqrt{N}$ extrapolation to the completed data set (of course, there is additional constraining power outside this redshift interval). These SDSS errors are augmented, primarily at high $k$, by adding a $\sqrt{10}$ reduced version of the $z \sim 3.9$ errors from McDonald et al. (2000), to represent about 20 high-resolution spectra (there are about 25 each in Songaila 2004 and Faucher-Giguère et al. 2008b, although not all of quite as high quality as McDonald et al. 2000). With this existing data in the redshift range $z=3.9-4.1$, compared to the theoretical difference between the models at $z=4$, one can distinguish formally between these two reionization models at $7 \sigma$ level. However, we note that the statistical differences between the two models are unmarginalized, i.e., not taking into account other physical effects or cosmological parameters that affect the Ly $\alpha$ flux power spectrum determination (e.g., McDonald et al. 2005a, 2005b). Therefore, the quoted statistical significance only serves as an indication of the potential power of these statistics, and the importance of accounting for this effect in more general fits to data. To determine the detectability of the effect after marginalization over all other parameters, one would need to assemble a complete model fitting apparatus as in McDonald et al. (2005b), including this effect, which is well beyond the scope of this Letter.

For comparison, the corresponding flux power spectrum ratios at $z=4$ and $z=5$, if both models follow the same EoS (given density), are shown (green curves) in Figure 2. In this case, aside from the small difference on small scales due to cumulative dynamical affects by the difference in the gas pressure histories, the two models have identical flux power spectrum on large scales. This demonstrates that the large difference in the flux power spectra between the two reionization models (black curves in Figure 2) is a result of differences in the contemporaneous temperature distributions.

\section{DISCUSSION}

The effect of inhomogeneous reionization on the flux power spectrum was explored by Lai et al. (2006) at $z=3$, based on a semianalytic model. Their focus was on $z=3$ and they found that, on large scales, $k \sim 0.001\left(\mathrm{~km} \mathrm{~s}^{-1}\right)^{-1}$, temperature fluctuations lead to an increase in the flux power spectrum by at most $10 \%$. Our focus is at higher redshifts $z=4-5$ and the effects, not surprisingly, are larger and potentially more discriminating.

A fluctuating radiation background, produced largely by radiation from sparsely distributed quasars but also by galaxies, can affect the flux power spectrum (Meiksin \& White 2004; Croft 2004; McDonald et al. 2005a). Larger fluctuations in the radiation background give rise to larger amplitudes of the flux power spectrum at large scales (e.g., McDonald et al. 2005a, Figures 6 and 7 therein). This enhancement of the flux power spectrum on large scales due to a fluctuating radiation background will be additional to what is caused by the gas temperature fluctuations shown here, if QSOs were dominant. The radiation contribution from stars may be more dominant at the redshift range of concern here (e.g., Faucher-Giguere et al. 2008a). Star formation is known to be biased, and hence, higher density regions, on average, tend to have higher radiation fields than lower density regions. Thus, the two effects due to a fluctuating radiation background and an inhomogeneous reionization process may be partially degenerate or have a tendency to cancel each 
other's contribution, although there is a possibility that the radiation fluctuations may be relatively modest (e.g., Mesinger \& Furlanetto 2009). A more careful modeling of the contribution from quasars as well as radiation sinks (such as Lyman limit systems) is required in a comprehensive modeling. The purpose of this Letter is to demonstrate that, if the effects on the Ly $\alpha$ flux power spectrum determination due to the epoch of reionization were the only relevant ones, then a precise measure of the flux power spectrum with the full SDSS I/II data would be able to place a very tight constraint on the epoch of reionization.

However, a detailed comparison between models and SDSS I/II observations requires a full analysis of all astrophysical/ cosmological processes that may affect the determination of the flux power spectrum, and some of them may be degenerate to varying degrees (McDonald et al. 2005a), including fluctuating radiation field, damped Ly $\alpha$ systems, galaxy formation feedback, initial photoheating temperature (i.e., related to initial mass function of high redshift galaxies), X-ray heating, He II reionization, among others, before its statistical potential can be precisely marginalized and quantified. We will perform such an analysis in a future study.

\section{CONCLUSIONS}

Utilizing state-of-the-art radiative transfer hydrodynamic simulations of cosmological reionization, we put forth the point that the inhomogeneous reionization process imprints important and quantitatively significant signatures in the IGM at $z=4.5 \pm 0.5$ that can be probed by the Ly $\alpha$ forest in the quasar absorption spectra. We illustrate that with $\operatorname{Ly} \alpha$ forest data only in the redshift range $z=3.9-4.1$ to be provided by the SDSS I/II full data sample, one can already distinguish between two cosmological epochs of reionization, one at $z=6$ and the other $z=9$ at $7 \sigma$ level, if they were the only effects on the determination of the Ly $\alpha$ flux power spectrum.

We thank J. Chang at NASA for invaluable supercomputing support. This work is supported in part by NASA grants NNG06GI09G and NNX08AH31G. Computing resources were in part provided by the NASA High-End Computing (HEC) Program through the NASA Advanced Supercomputing (NAS) Division at Ames Research Center. P.M. acknowledges support of the Beatrice D. Tremaine Fellowship. H.T. is supported by an Institute for Theory and Computation Fellowship.

\section{REFERENCES}

Barkana, R., \& Loeb, A. 2001, Phys. Rep., 349, 125

Becker, R. H., Fan, X., White, R. L., Strauss, M. A., Narayanan, V. K., Lupton, R. H., Gunn, J. E., \& Annis, J. 2001, AJ, 122, 2850

Becker, G. D., Rauch, M., \& Sargent, W. L. W. 2007, ApJ, 662, 72

Cen, R. 2003, ApJ, 591, 12

Cen, R., \& McDonald, P. 2002, ApJ, 570, 457

Croft, R. A. C. 2004, ApJ, 610, 642

Dunkley, J., et al. 2009, ApJS, 180, 306

Fan, X., Carilli, C. L., \& Keating, B. 2006a, ARA\&A, 44, 415

Fan, X., et al. 2001, AJ, 122, 2833

Fan, X., et al. 2006b, AJ, 132, 117

Faucher-Giguere, C., Lidz, A., Zaldarriaga, M., \& Hernquist, L. 2008a, ApJ, 682, L9

Faucher-Giguère, C.-A., Prochaska, J. X., Lidz, A., Hernquist, L., \& Zaldarriaga, M. 2008b, ApJ, 681, 831

Furlanetto, S. R., \& Oh, S. P. 2009, ApJ, 701, 94

Furlanetto, S. R., Zaldarriaga, M., \& Hernquist, L. 2004, ApJ, 613, 1

Haiman, Z., \& Holder, G. P. 2003, ApJ, 595, 1

Hui, L., \& Gnedin, N. Y. 1997, MNRAS, 292, 27

Hui, L., \& Haiman, Z. 2003, ApJ, 596, 9

Iliev, I. T., Mellema, G., Pen, U.-L., Merz, H., Shapiro, P. R., \& Alvarez, M. A. 2006, MNRAS, 369, 1625

Komatsu, E., et al. 2009, ApJS, 180, 330

Lai, K., Lidz, A., Hernquist, L., \& Zaldarriaga, M. 2006, ApJ, 644, 61

Lee, K.-G., Cen, R., Gott, J. R. I., \& Trac, H. 2008, ApJ, 675, 8

McDonald, P., Miralda Escudé, J., Rauch, M., Sargent, W. L. W., Barlow, T. A., Cen, R., \& Ostriker, J. P. 2000, ApJ, 543, 1

McDonald, P., Seljak, U., Cen, R., Bode, P., \& Ostriker, J. P. 2005a, MNRAS, 360, 1471

McDonald, P., et al. 2005b, ApJ, 635, 761

McDonald, P., et al. 2006, ApJS, 163, 80

Meiksin, A., \& White, M. 2004, MNRAS, 350, 1107

Mesinger, A., \& Furlanetto, S. 2009, arXiv:0906.3020

Songaila, A. 2004, AJ, 127, 2598

Theuns, T., Schaye, J., Zaroubi, S., Kim, T.-S., Tzanavaris, P., \& Carswell, B. 2002, ApJ, 567, L103

Trac, H., Cen, R., \& Loeb, A. 2008, ApJ, 689, L81

Wyithe, J. S. B., \& Cen, R. 2007, ApJ, 659, 890 